\title{
Tiempo requerido de enfermería para un cuidado con calidad
}

\author{
Ana Margarita Puerta Cataño² \\ Dora Lucía Gaviria Noreña \\ Sandra Lorena Duque Henao ${ }^{4}$
}

doi:10.11144/Javeriana.ie19-2.trec

Cómo citar: Puerta Cataño AM, Gaviria Noreña DL, Duque Henao SL. Tiempo requerido de enfermería para un cuidado con calidad. Investig Enferm Imagen Desarr. 2017;19(2):14559. http://dx.doi.org/10.11144/Javeriana.ie19-2.trec

1. Artículo original de investigación. Fecha de recepción: 15 de diciembre de 2015. Fecha de aceptación: 14 de junio de 2016.

2. Enfermera. Magíster en Salud Pública. Magíster en Educación. Docente de la Facultad de Enfermería de la Universidad de Antioquia, Medellín, Colombia. Correo electrónico: nisaba1@hotmail.com

3. Enfermera. Magíster en Salud Colectiva. Especialista en Epidemiología. Docente de la Facultad de Enfermería de la Universidad de Antioquia, Medellin, Colombia. Correo electrónico: dora.gaviria@udea.edu.co,doralgaviria@gmail.com

4. Enfermera. Magíster en Epidemiología. Docente de la Facultad de Enfermería, Universidad de Antioquia. Correo electrónico: lorduq@gmail.com 


\section{Resumen}

Objetivo: Identificar el tiempo requerido de enfermería para el cuidado, la educación, la investigación y la gestión del cuidado y del servicio, a fin de determinar el talento humano necesario para la prestación del cuidado con calidad en un hospital de primer nivel de atención. Método: Estudio descriptivo-transversal realizado con estudiantes de enfermería del último semestre de pregrado. Se aplica el proceso de atención de enfermería (PAE), siguiendo los criterios de la NANDA y la valoración con cada sujeto de cuidado. Desde los diagnósticos y las intervenciones de enfermería se determina el tiempo para el cuidado, se utiliza la técnica de análisis por consenso desde la evidencia para calcular el tiempo para la investigación, la educación, la gestión del cuidado y del servicio. Resultados: Se identifican 182 diagnósticos de enfermería con sus intervenciones y el respectivo tiempo para el cuidado, por paciente y por profesional y auxiliar de enfermería en el servicio de hospitalización. También se evidencia el tiempo para las funciones que el personal de enfermería debe realizar que no son cuidado directo, pero que facilitan el logro de objetivos y ayudan al ideal de un cuidado con calidad y centrado en el sujeto del cuidado. Se elaboran 68 guías de enfermería para la unificación de criterios. Conclusiones: Calcular el talento humano de enfermería debe partir de las necesidades de los sujetos de cuidado en su contexto y de la estimación del tiempo para la gestión del cuidado y del servicio, así como para la educación e investigación.

Palabras clave: atención de enfermería; atención dirigida al paciente; planificación de atención al paciente

\section{Nursing Time Required for Quality Care}

\section{Abstract}

Objective: To identify the nursing care time, education, research and care management and service, to determine the human talent needed for the provision of quality care at a hospital in primary care. Method: descriptive cross-sectional study with last semester nursing students. The nursing care plan (NCP) is applied, following the criteria from the NANDA and an assessment is carried with each care subject. The time for care is determined from the diagnoses and nursing interventions; the consensus methodology for analyzing evidence is used to calculate the time for research, education, care management and service. Results: 182 nursing diagnoses were identified with its interventions and their respective time care per patient, professional, and auxiliary nursing inpatient service. There is also evidence of time for functions that nurses should carry out which are not direct care, but which facilitate the achievement of goals and help the ideal of quality and subject-centered care .68 nursing guidelines for the unification of criteria were developed. Conclusions: Calculating nursing human talent must emerge from the needs of the care subjects in their context, from time estimation for care management and service, as well as for education and research.

Keywords: nursing care; patient-centered care; patient care planning 


\section{Tempo precisado de enfermagem para o cuidado de qualidade}

\section{Resumo}

Objetivo: Identificar o tempo precisado de enfermagem para o cuidado, educação, pesquisa e gestão do cuidado e serviço, a fim de determinar o talento humano necessário para a prestação de cuidado com qualidade em um hospital de primeiro nível de atenção. Metodo: Estudo descritivo-transversal realizado com estudantes de enfermagem de último semestre de formação. O processo de atenção de enfermagem (PAE) é aplicado de acordo com critérios da NANDA e a valoração com cada sujeito de cuidado. Desde os diagnósticos e as intervenções de enfermagem determina-se o tempo para o cuidado, usando a técnica de análise por consenso desde a evidência para calcular o tempo para pesquisa, ensino, gestão do cuidado e serviço. Resultados: Foram identificados 182 diagnósticos de enfermagem com suas intervenções e o respectivo tempo para o cuidado, por paciente e por profissional e auxiliar de enfermagem no serviço de hospitalização. Mesmo foi evidenciado o tempo para as funções que o pessoal de enfermagem deve realizar diferentes a cuidado direito, mas que facilitam a consecução de objetivos e ajudam no ideal de um cuidado de qualidade e centrado no sujeito do cuidado. Foram elaboradas 68 guias de enfermagem para a unificação de critérios. Conclusões: Calcular o talento humano de enfermagem deve partir das necessidades dos sujeitos do cuidado no seu contexto e a estimação do tempo para a gestão do cuidado e serviço, assim como para ensino e pesquisa.

Palavras-chave: atendimento em enfermagem; atenção dirigida ao paciente; planejamento de atenção ao paciente 


\section{Introducción}

Brindar un cuidado de excelencia y centrado en el ser humano requiere especificar en número y en cualificación el personal de enfermería que atenderá a los sujetos de cuidado, según los avances científicos, tecnológicos y disciplinares y según las exigencias del contexto. Al respecto, el Consejo Internacional de Enfermeria, en su documento Personal fiable vidas salvadas (1), reconoce que la dotación de personal de enfermería adecuado para dispensar cuidados totales favorece la calidad, disminuye la mortalidad y las complicaciones del sujeto del cuidado y aumenta su satisfacción y la del personal de enfermería; además, evidencia la contención de costos en la prestación de los servicios de salud.

En cuanto a lo legal y la responsabilidad deontológica: contar con el personal de enfermería adecuado en los servicios de salud es un requisito de calidad para la práctica del profesional de enfermería en Colombia, lo plantea la Ley 266 de 1996 (2) y la Ley 911 de 2004 (3), las cuales determinan que el servicio de enfermería debe ser oportuno, sin riesgos y acorde con los estándares de cuidado según los niveles de atención.

Ahora, estudios de Aiken y cols. (4-7) muestran la asociación entre un número adecuado de enfermeras con alta cualificación con índices de mortalidad más bajos, mayor efectividad terapéutica y reducción de eventos adversos. En Colombia, la atención en salud (8) está orientada por la calidad y la seguridad, sustentadas en legislación y politicas institucionales; pero para que se implemente es obligación que el cuidado de la salud de los individuos y los colectivos esté a cargo de personal cualificado y en número suficiente para cumplir con los requerimientos y satisfacer las necesidades de esos sujetos de cuidado implicados.

En cuanto a calidad, enfermería $(2,3)$ tiene una misión relacionada con el bienestar, la defensa de los derechos de los sujetos de cuidado y la protección del gremio, en lo pertinente a prevenir la sobrecarga laboral y el estrés en el trabajo, como condiciones ideales para garantizar un cuidado humano centrado en el otro y libre de riesgos.

El cuidado de enfermería con calidad incluye: una dimensión ética, que se refiere a los aspectos de la relación interpersonal, es decir, el establecimiento de la empatía y el respeto a la dignidad humana, en contextos culturales diferentes; una dimensión científica y tecnológica, que comprende la prestación del cuidado pertinente según los avances científicos y técnicos disciplinares; y una dimensión perceptiva, que incluye la capacidad de comprender las potencialidades y necesidades del sujeto de cuidado y las formas para satisfacerlas.

Ahora, en las instituciones es común encontrar el proceso de cálculo de personal de enfermería gestionado desde la aplicación de indicadores foráneos, con resultados no ajustados al contexto y a las necesidades, lo que genera un desequilibrio entre la demanda de servicios de los sujetos de cuidado y la oferta de personal. Es aún más grave cuando el desacierto no se corrige y el personal tiende a desplazar el cuidado para cumplir con otras funciones administrativas de gestión del servicio, las cuales dejan oculto e invisible el cuidado prestado por el profesional de enfermería. 
Igual situación de desbalance se presenta cuando para la asignación del equipo humano de enfermería se utiliza el método Delfos, por medio del cual el consenso del juicio de expertos se realiza desde el recuerdo de estos, sin evidencias objetivas que muestren la carga de trabajo diaria del personal de enfermería en comparación con los requerimientos de los pacientes, la gestión del cuidado, la gestión del servicio de enfermería, así como otras funciones asignadas por las directivas o asumidas por la costumbre en la dinámica institucional. Algunos estudios sobre carga de trabajo diario en enfermería han evidenciado cómo el personal de la salud de esta área tienen la mayor incidencia del sindrome de desgaste profesional o burnout (9).

Surge la pregunta: ¿es posible identificar el talento humano de enfermería requerido para el cuidado de calidad desde la práctica cotidiana y calcular el tiempo real del cuidado, la educación, la investigación, la gestión del cuidado y del servicio en una institución de primer nivel de atención?

\section{Método}

Este fue un estudio descriptivo-transversal que integró en diferentes momentos el tiempo requerido para el cuidado con información contextualizada, según las formas de trabajo del personal, la situación de los pacientes y la complejidad del servicio.

La información se obtuvo durante el tiempo de práctica del curso Gestión de Enfermería II, por la docente y algunos estudiantes de último semestre del pregrado de Enfermería de la Universidad de Antioquia. El tiempo de duración fue de seis semestres entre el 2010 y el 2013 en un el servicio de hospitalización de una institución de primer nivel de atención (10-15).

Se aplicó el método de Puerta Cataño (16), "Planes de dotación desde el cuidado, una utopía alcanzable"; así mismo, el trabajo tiene la influencia de Whetsell (17), quien afirma:

[...] es importante que los diagnósticos de enfermería sean ajustados desde la realidad viviente de las personas en las diferentes regiones y países [...] las enfermeras deben realizar sus propios Diagnósticos de Enfermería basados en las situaciones que viven las personas a las que cuidan, no son los mismos diagnósticos en tiempo y en lugar realizados por los estadounidenses, los que se podrían aplicar en la región suramericana propiamente la colombiana, puesto que se cuentan con situaciones culturales y comportamentales propias de esta región, por ello nosotros esperamos que los enfermeros colombianos nos den sus propios Diagnósticos de Enfermería.

Bajo la técnica del juicio de expertos se validó la información, para comprender, analizar e identificar el tiempo requerido para el cuidado, desde diagnósticos de enfermería obtenidos, y el seguimiento a las funciones realizadas lo llevaron a cabo los expertos, quienes con su experiencia tenían la información más cercana a sus realidades cotidianas. La aplicación del proceso de enfermería en el contexto favorece el análisis desde la evidencia y no desde la subjetividad o el recuerdo; además, los resultados de docentes 
y estudiantes son conocidos y socializados diariamente con el profesional de enfermería, como experto, encargado del servicio de hospitalización (4). En este sentido, los estudios en el área del diagnóstico de enfermería, según Fortín, citado en Carrillo González y cols. (18), buscan la validación de contenido "como la representatividad del conjunto de enunciados que constituyen el concepto que se va a medir y las observaciones de los expertos acerca de las características en una situación". Con lo anterior se establece la validez del método desde el proceso de atención en enfermería (PAE), como el camino para el desarrollo disciplinar y profesional.

En un primer momento, durante quince semanas por semestre, de lunes a viernes y en el horario de práctica de los estudiantes, se recolectó la información sobre el tiempo requerido para el cuidado de los pacientes hospitalizados en esos días de práctica. A partir del PAE con los sujetos de cuidado se identificaron los diagnósticos de enfermería e intervenciones, y con ellos se construyó una matriz donde se registró y consolidó la información del tiempo y número de veces requerido para cada una de las intervenciones por diagnóstico de enfermería, además de clasificarla por categoría de personal, es decir, si dicha actividad la realiza el profesional de enfermería o con la participación de los auxiliares de enfermería. Posteriormente, la información se socializó con el profesional de enfermería jefe del servicio, como experto, y se hicieron los ajustes.

Es de anotar que la información construida en el servicio de hospitalización se hace desde la interacción con los sujetos de cuidado que durante el periodo están hospitalizados en la misma institución; ahora, en todo el estudio se siguieron como directrices los criterios de la North American Nursing Diagnosis (NANDA), que respetan las particularidades y la valoración realizada con cada uno de los pacientes participantes.

Para el cálculo del tiempo requerido en la gestión, educación e investigación se utilizaron instrumentos construidos desde el análisis de las competencias y funciones de enfermería contempladas en los perfiles de cargo institucionales. Se completaron con información obtenida por observación de los funcionarios por categoría profesional y auxiliar en el servicio de hospitalización. Dichos formatos se llevaron a una prueba piloto y se validaron, a través del método de consenso de expertos, con los profesionales de enfermería que, en su momento, eran los jefes del servicio de hospitalización. En los diferentes semestres se obtuvo el consentimiento informado de los sujetos de cuidado y del personal de enfermería implicado.

La información se recolectó desde el 2010 al 2013, como un proceso continuo que ha favorecido la construcción de instrumentos y la validación de contenidos desde el juicio de expertos y la vinculación de la reflexión desde estudiantes y docentes de la práctica de gestión. En este sentido, la elaboración de cada instrumento obedeció a la observación participante, al análisis y a la validación con los profesionales de enfermería. 


\section{Resultados}

Se identificaron 182 diagnósticos de enfermería con sus respectivas acciones de cuidado y 68 funciones que permiten o facilitan la gestión de cuidado y del servicio, además del tiempo requerido en cada una de ellas y por categoría profesional o auxiliar.

En la tabla 1, a manera de ejemplo, se presenta uno de los diagnósticos con las intervenciones de enfermería e incluye el número de veces que estas se realizan por día y por categoría de personal de enfermería estipulada en Colombia, por la Ley 266 de 25 de enero de1996. Este método evidencia la realidad de los cuidados de enfermería desde la situación de los pacientes y el tiempo establecido por consenso a partir de la realidad observada, a la vez que aporta hechos para elaborar indicadores, pues al utilizar información real actualizada y estandarizada, permite sistematizar y evaluar la prestación del cuidado de enfermería.

TABla 1. Tiempo en minutos por día para el cuidado, según diagnósticos de enfermería y categoría de personal, servicio de hospitalización ESE hospital primer nivel, 2012

\begin{tabular}{|c|c|c|c|c|c|c|c|}
\hline \multirow{3}{*}{ 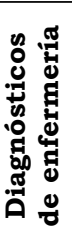 } & \multirow{3}{*}{$\begin{array}{l}\text { Intervenciones de } \\
\text { enfermeria }\end{array}$} & \multicolumn{4}{|c|}{ Categoria } & \multirow{2}{*}{\multicolumn{2}{|c|}{ Total }} \\
\hline & & \multicolumn{2}{|c|}{ Enfermera } & \multicolumn{2}{|c|}{$\begin{array}{l}\text { Auxiliar de } \\
\text { enfermeria }\end{array}$} & & \\
\hline & & $\begin{array}{c}\text { Tiempo } \\
\text { (min) }\end{array}$ & $\begin{array}{l}\text { Núm. } \\
\text { veces }\end{array}$ & $\begin{array}{c}\text { Tiempo } \\
\text { (min) }\end{array}$ & Veces & $\begin{array}{l}\text { Enfer- } \\
\text { mera }\end{array}$ & $\begin{array}{l}\text { Auxiliar } \\
\text { enfer- } \\
\text { meria }\end{array}$ \\
\hline \multirow{11}{*}{ 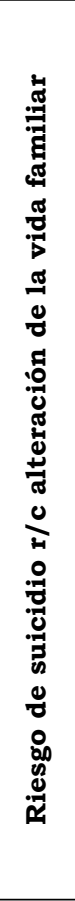 } & $\begin{array}{l}\text { Establecer una relación } \\
\text { terapéutica }\end{array}$ & 20 & 1 & 8 & 1 & 20 & 8 \\
\hline & $\begin{array}{l}\text { Retirar del entorno los objetos } \\
\text { que representen un peligro }\end{array}$ & - & - & 7 & 1 & - & 7 \\
\hline & $\begin{array}{l}\text { Indagar sobre las relaciones y } \\
\text { dinámicas familiares }\end{array}$ & 8 & 1 & 5 & 1 & 8 & 5 \\
\hline & $\begin{array}{l}\text { Explorar las causas especifi- } \\
\text { cas de intento de suicidio }\end{array}$ & 10 & 1 & - & - & 10 & - \\
\hline & $\begin{array}{l}\text { Ofrecer información sobre } \\
\text { diferentes redes de apoyo }\end{array}$ & 8 & 1 & 4 & 1 & 8 & 4 \\
\hline & $\begin{array}{l}\text { Analizar con el equipo } \\
\text { interdisciplinario los } \\
\text { hallazgos de valoración } \\
\end{array}$ & 5 & 1 & - & - & 5 & - \\
\hline & Gestionar proceso de remisión & 15 & 1 & 10 & 1 & 15 & 10 \\
\hline & $\begin{array}{l}\text { Valorar la respuesta ante } \\
\text { la terapia analgésica, así } \\
\text { como la aparición de efectos } \\
\text { secundarios }\end{array}$ & 2 & 2 & 2 & 4 & 4 & 8 \\
\hline & $\begin{array}{l}\text { Brindar educación al paciente } \\
\text { y su familia acerca de los } \\
\text { cuidados necesarios }\end{array}$ & 5 & 1 & - & - & 5 & - \\
\hline & $\begin{array}{l}\text { Realizar curación según } \\
\text { indicaciones }\end{array}$ & 15 & 1 & - & - & 15 & - \\
\hline & Mantener la ropa limpia y seca & - & - & 3 & 2 & - & 6 \\
\hline
\end{tabular}

Fuente: Puerta AM, Giraldo A, Mosquera H, Preciado L, Tabares D. Diagnósticos en enfermería más frecuente en el servicio de hospitalización de la ESE hospital primer nivel; 2012. 
Se identificó el tiempo requerido por categoría de enfermería para realizar las funciones de gestión del cuidado (tabla 2).

TABla 2. Promedio de tiempo en minutos requerido por vez, para algunas funciones de gestión del cuidado, por paciente y por categoría. ESE hospital de primer nivel, 2012

\begin{tabular}{|l|r|r|}
\hline \multirow{2}{*}{\multicolumn{1}{|c|}{ Funciones }} & \multicolumn{2}{c|}{ Tiempo promedio en minutos } \\
\cline { 2 - 3 } & \multicolumn{1}{c|}{ Enfermera } & \multicolumn{1}{c|}{ Auxiliar } \\
\hline Recibo y entrega de turno & 5 & 5 \\
\hline Ronda de enfermería & 14 & - \\
\hline Ronda con equipo de salud & 3 & - \\
\hline Aplicar PAE & 33 & - \\
\hline Hacer ingreso & 13 & 6 \\
\hline Realizar egreso & 14 & 7 \\
\hline Ronda educativa por equipo de salud & 10 & - \\
\hline Dirigir el cuidado & 6 & - \\
\hline Solicitar evaluaciones de ayudas diagnósticas & 2 & - \\
\hline Dar educación: aspectos hospitalarios & 7 & 6 \\
\hline Educar cuidador en casa & 10 & - \\
\hline Solicitar medicamentos & 3 & - \\
\hline Total & 120 & 24 \\
\hline
\end{tabular}

PAE: proceso de atención de enfermería.

Fuente: Puerta AM, Pérez S. Tiempo requerido por día para la gestión del cuidado y para las intervenciones del cuidado, desde el proceso de atención de enfermería, de algunos pacientes internados en un primer nivel; 2012.

Se monitoreó el tiempo de personal de enfermería para realizar las acciones de apoyo al cuidado y para el funcionamiento del servicio de hospitalización, acciones que si bien la mayoría no se hacen directamente con el paciente, traen un beneficio para este o incluye cuidados para su ambiente o complementan y apoyan las acciones de cuidado directo. En el trabajo se rastrearon 30 funciones. En la tabla 3 se presentan 9 a manera de ejemplo.

TABLA 3. Tiempo requerido por categoría de personal de enfermería para realizar funciones de apoyo al cuidado y para el funcionamiento del servicio de hospitalización. ESE hospital de primer nivel, 2013

\begin{tabular}{|l|r|r|r|r|}
\hline \multirow{2}{*}{ Funciones } & \multicolumn{4}{|c|}{ Tiempo en minutos por vez } \\
\cline { 2 - 5 } & Enfermera & Auxiliar & Secretaria & Otros \\
\hline $\begin{array}{l}\text { Asistir al paciente en la revisión } \\
\text { médica }\end{array}$ & 7,07 & - & - & - \\
\hline $\begin{array}{l}\text { Hacer pedidos de almacén, } \\
\text { medicamentos y material médico- } \\
\text { quirúrgico }\end{array}$ & 15,5 & - & - & - \\
\hline $\begin{array}{l}\text { Supervisar o presenciar la entrega } \\
\text { de pedidos }\end{array}$ & 9,57 & 4,75 & - & - \\
\hline $\begin{array}{l}\text { Revisar equipos de uso diario y } \\
\text { para mantenimiento preventivo }\end{array}$ & 11,8 & - & - & - \\
\hline
\end{tabular}




\begin{tabular}{|l|r|r|r|r|}
\hline $\begin{array}{l}\text { Revisar y actualizar historias } \\
\text { clinicas }\end{array}$ & 11,5 & 6,5 & - & - \\
\hline Elaborar y actualizar kárdex & 5,7 & - & - & - \\
\hline $\begin{array}{l}\text { Elaborar planes de cuidado y } \\
\text { registrar en la historia }\end{array}$ & 12,9 & - & - & - \\
\hline Atender demanda inducida & 2,8 & 3,5 & - & - \\
\hline $\begin{array}{l}\text { Organizar estantes de material y } \\
\text { equipo }\end{array}$ & - & 4,79 & - & - \\
\hline $\begin{array}{l}\text { Revisar el equipo de emergencias } \\
\text { (carro de paros) en salas de partos } \\
\text { y hospitalización }\end{array}$ & 8,6 & - & - & - \\
\hline
\end{tabular}

Fuente: Puerta AM, Buitrago L, Gallego D. Tiempo requerido por categoría de personal de enfermería para realizar funciones de apoyo al cuidado y para el funcionamiento del servicio de hospitalización. ESE hospital primer nivel, 2013.

Referente a las funciones de gestión, educación e investigación, se identificaron 68 con numerosas actividades, tareas que requieren una frecuencia diaria, mensual y anual según su complejidad y alcance. A manera de ejemplo, en la tabla 4 se evidenciaron, desde la gestión del servicio, 10 funciones y 38 actividades, con sus respectivos tiempos y momentos que dimensionan la necesidad del talento humano de enfermería para lograr un cuidado con calidad, sin tener que apropiarse del tiempo para el cuidado directo.

TABla 4. Tiempo en minutos requerido de profesional de enfermería para realizar algunas funciones relacionadas con la gestión del servicio de hospitalización, investigación y educación. ESE hospital de primer nivel; 2013

\begin{tabular}{|c|c|c|c|c|}
\hline \multirow{2}{*}{ Funciones } & \multicolumn{3}{|c|}{ Minutos por } & \multirow{2}{*}{$\#$} \\
\hline & Vez & Mes & Año & \\
\hline \multicolumn{5}{|c|}{ Dirigir el personal a cargo } \\
\hline Explicar el trabajo por paciente & 5 & - & - & 1 \\
\hline Explicar el trabajo por paciente aislado & 5 & - & - & 1 \\
\hline Explicar el trabajo por paciente urgente & 5 & - & - & 1 \\
\hline Explicar funciones de apoyo al cuidado & 6 & - & - & 1 \\
\hline Dar instrucciones antes de ausentarse del servicio & 15 & - & - & 1 \\
\hline Inducción al personal nuevo & 60 & - & - & 1 \\
\hline Entrenamiento del personal & 45 & - & - & 1 \\
\hline Trabajo específico de informática & 6 & - & - & 1 \\
\hline \multicolumn{5}{|c|}{ Participar en rondas de: } \\
\hline Enfermería & 7 & - & - & 2 \\
\hline (Equipo) Necesidades farmacológicas del paciente & 7 & - & - & 1 \\
\hline (Equipo) Necesidades educativas del paciente & 15 & - & - & 1 \\
\hline \multicolumn{5}{|c|}{ Realizar asignación de pacientes y actividades: } \\
\hline Organizar las asignaciones & 10 & - & - & 1 \\
\hline $\begin{array}{l}\text { Realizar reasignaciones por incapacidades y } \\
\text { calamidades }\end{array}$ & 12 & - & - & 1 \\
\hline Realizar reasignaciones por remisión de pacientes & 7 & - & - & 1 \\
\hline Realizar el diagnóstico necesidades del servicio & - & - & - & - \\
\hline
\end{tabular}




\begin{tabular}{|c|c|c|c|c|}
\hline Por semana & 30 & & - & 1 \\
\hline Por mes: aplicar la matriz DOFA & - & 120 & - & 1 \\
\hline Elaborar el plan de horarios para el personal & - & 480 & - & 1 \\
\hline Proponer el plan de vacaciones para enfermería & - & - & 90 & 1 \\
\hline Proponer plan desarrollo del personal enfermería & - & - & 90 & 1 \\
\hline Proponer plan de acción afín con enfermería & - & - & 192 & 1 \\
\hline \multicolumn{5}{|c|}{ Enseñar derechos y deberes } \\
\hline Adulto mayor y familiar & 8 & - & - & 1 \\
\hline Gestante y familiar & 6 & - & - & 1 \\
\hline Madres de niños pequeños & 5 & - & - & 1 \\
\hline Pacientes en estado de aislamiento & 8 & - & - & 1 \\
\hline Adulto & 8 & - & - & 1 \\
\hline \multicolumn{5}{|c|}{ Participa en investigaciones autorizadas sobre: } \\
\hline Dispositivos médicos & 180 & - & - & 1 \\
\hline Maternidad segura & 180 & - & - & 1 \\
\hline Cáncer de mama & 180 & - & - & 1 \\
\hline Paciente diabético & 180 & - & - & 1 \\
\hline Clínica de heridas & 180 & - & - & 1 \\
\hline Participa actividades proceso de acreditación & 120 & - & - & 1 \\
\hline \multicolumn{5}{|c|}{ Realiza informes sobre: } \\
\hline Reunión de enfermería & 40 & - & - & 1 \\
\hline Situaciones administrativas & 15 & - & - & 1 \\
\hline Contra referencias & 10 & - & - & 1 \\
\hline \multicolumn{5}{|c|}{ Realiza auditorias de historias clinicas } \\
\hline En hospitalización & 7 & - & - & 1 \\
\hline En urgencias & 10 & - & - & 1 \\
\hline Por procedimientos & 10 & - & - & 1 \\
\hline \multicolumn{5}{|c|}{ Realizar auditoria de enfermeria } \\
\hline Verificar escalas de Braden, por historia & 4 & - & - & 1 \\
\hline Formato: paciente gestor & 3 & - & - & 1 \\
\hline
\end{tabular}

Fuente: Puerta AM, Montoya J, Valencia Y. Tiempo requerido de profesional de enfermería para realizar algunas funciones relacionadas con la gestión del servicio de hospitalización, investigación y educación. ESE hospital de primer nivel; 2013.

\section{Discusión}

En los círculos estatales, académicos, institucionales y gremiales se habla de la obligación de asegurar una atención de enfermería excelente, humana, segura y centrada en las necesidades del paciente, lo cual se respalda en la Ley 266 del 25 de enero de 1996 (2), en su artículo 20, que reza: "son deberes del profesional de enfermería entre otros 1 . Brindar atención integral de enfermería de acuerdo a los principios generales y específicos de su práctica establecidos en esta Ley [...] 2. Velar por que se brinde atención profesional de enfermería de calidad, a todas las personas y comunidades sin distinción”.

Por lo tanto, el profesional de enfermería debe garantizar un método de cuidado que le permita integrar todas las bases científicas, metodológicas, éticas y legales. La respuesta a ese requerimiento se encuentra en el PAE, 
particularmente en los diagnósticos de enfermería con sus intervenciones, aplicados en la cotidianidad del cuidado formal. Una conclusión lógica de esta postura puede ser: si se determina prestar un cuidado con calidad, se debe asegurar tiempo de personal de enfermería por categoría para realizar el PAE, los diagnósticos de enfermería y las intervenciones de cuidado que los pacientes requieren.

La misma Ley 266, en el artículo 17, entre otras, señala las siguientes competencias de los profesionales de enfermería:

[...] 3. Definir y aplicar los criterios y estándares de calidad en las dimensiones éticas, científicas y tecnológicas de la práctica de enfermería. 4. Dirigir los servicios de salud y de enfermería. [...] 6. Ejercer responsabilidades y funciones de asistencia, gestión, administración, investigación, docencia, tanto en áreas generales como especializadas y aquellas conexas con la naturaleza de su ejercicio, tales como asesorías, consultorias y otras relacionadas.

Lo mencionado hace pensar que si se establece dar un cuidado con calidad, se debe asegurar tiempo de personal de enfermería, por categoría, para realizar todas las funciones que manda la ley en este artículo.

El artículo 21 indica entre los derechos del profesional de enfermería: “6. Contar con los recursos humanos y materiales necesarios y adecuados para cumplir con sus funciones de manera segura y eficaz, que le permitan atender dignamente a quien recibe sus servicios. [...] 8. Tener derechos a condiciones de trabajo que aseguren una atención de enfermería de calidad para toda la población".

Cuando enfermería habla de cuidar, se refiere al desarrollo de las actividades que permiten alcanzar un mayor grado de bienestar, de desarrollo que implica conocer al ser humano que padece la enfermedad, en medio de un acto de interacción mediado por la comunicación, los valores éticos y los conocimientos científicos como un verdadero encuentro con el otro, con su grupo familiar y con el contexto social donde este vive.

Se cuida cuando hay atención directa con el sujeto de cuidado durante la hospitalización; pero también se cuida cuando se educa, así como cuando se gestionan recursos para el cuidado y el funcionamiento del servicio. Sin embargo, el personal de enfermería ha estado sometido al trámite administrativo, de facturación, de secretariado y de vigilancia, que lo alejan del sujeto de cuidado y de su verdadera esencia profesional. Estudios de dotación de personal de enfermería, de carga laboral y de condiciones de trabajo han demostrado que solo se logra la excelencia en el cuidado si se cuenta con personal adecuado, idóneo y satisfecho. Como se evidencia en los estudios para la creación de escalas como la Nursing Activities Score, de Miranda y cols. (19), Grillo y cols. (20) y Conishi (21). Además, el artículo 19 de la Ley 266 de 1996 (2), que reglamenta que con

[...] el fin de asegurar un cuidado de enfermería de calidad científica, técnica, social, humana y ética se cumplirán las siguientes disposiciones: 
1. El ejercicio de la profesión de enfermería en Colombia se ejercerá dentro de los criterios y normas de calidad y atención y de educación que establezca la Asociación Nacional de Enfermeras de Colombia, ANEC y la Asociación Colombiana de Facultades de Enfermería, Acofaen, en concordancia con lo definido por el Consejo Técnico Nacional de Enfermería y lo establecido por los Organismos gubernamentales. [...] 5. Los profesionales de enfermería vigilarán la conformación cualitativa y cuantitativa de los recursos humanos de enfermería que requieran las instituciones de salud y los centros de enfermería para su funcionamiento de acuerdo a los criterios y normas establecidas por el Consejo Técnico Nacional de Enfermería.

Algo más, el parágrafo de este artículo manifiesta: "Las disposiciones para el cálculo de personal de enfermería, estarán basadas en normas nacionales e internacionales que tengan en cuenta el estado de salud de los usuarios, que demanden mayor o menor tiempo de atención de enfermería” (2).

En cuanto a responsabilidad institucional, corresponde a la dirección del hospital la asignación de los tiempos de personal de enfermería, para cubrir las necesidades de cuidado y la realización de todas las funciones para que la organización de enfermería actúe sin traumatismo.

Ahora, la dotación del personal de enfermería debe estar basada en las necesidades reales y potenciales de los sujetos de cuidado, identificadas a través de la valoración del profesional de enfermería y de la selección de intervenciones encaminadas a dar respuesta a estas necesidades, con fundamentos conceptuales, metodológicos disciplinares la evidencia de la experiencia del quehacer profesional.

La reflexión de la práctica favorece el desarrollo de habilidades y competencias para brindar un cuidado humanístico y de calidad. Como Benner (22) lo expresa en su obra From Novice to Expert: Excellence and Power in Clinical Nursing Practice, el conocimiento integrado en la práctica del oficio de la enfermera proporciona la adquisición de habilidades y experticia frente a las funciones del cuidado, a la gerencia eficaz, a la investigación y a una práctica basada en la excelencia y en la ética reflexiva.

Además, porque el profesional de enfermería es quien tiene la capacidad para asumir la elaboración de la propuesta para la dotación de personal de enfermería, ya que conoce el diagnóstico de enfermería de cada paciente, el plan de cuidados individualizado, con sus respectivas intervenciones; además, conoce los protocolos, las guías y los programas de la institución.

A lo anterior se le puede adicionar que el profesional de enfermería es quien tienen claro la realidad institucional y del contexto; por eso sabe, aparte de los requerimientos de tiempo para el cuidado directo, la necesidad de tiempo para las funciones de apoyo al cuidado, tiempo requerido para la gestión del cuidado, para la investigación, para la educación a los pacientes y al personal, además de la gestión del servicio de enfermería, como lo contempla la Ley 266 de 1996.

El profesional de enfermería está llamado a asumir el liderazgo para elaborar los estudios de dotación de personal, argumentados desde la 
experiencia y la investigación, donde se tenga en cuenta la realidad vivida, recogida desde el quehacer cotidiano, procesada y argumentada en una transformación que integre todos los requerimientos para dar un cuidado con calidad, seguro y libre de riesgos, como manda la ley y las instituciones.

Siguiendo a Leninger (23), la meta del profesional de enfermería es brindar cuidados pertinentes y oportunos a los individuos y colectivos mediante una identificación de sus necesidades, apoyados en el proceso de enfermería para restablecer la independencia y autonomía, promover la integridad biopsicosocial y mantener la capacidad de los individuos y colectivos de autocuidado.

Finalmente, con la elaboración de esos estudios en las instituciones y en las regiones, se tienen por escrito las evidencias de la actividad continua de los profesionales de enfermería desde el PAE, así como los diagnósticos e intervenciones de enfermería, lo que en el tiempo permite consolidar el quehacer de la profesión desde la realidad de los sujetos de cuidado en el contexto local y regional y, por qué no, nacional. Aquí se espera que las agremiaciones y el Consejo Técnico Nacional de Enfermería tengan su protagonismo para buscar una estandarización de los cuidados y del tiempo requerido para el trabajo de enfermería desde las necesidades del cliente interno, externo y desde las mismas instituciones.

\section{Conclusiones}

La planeación del talento humano en enfermería y la determinación del tiempo requerido para el cuidado con calidad deben basarse en las necesidades de los sujetos de cuidado en una forma objetiva, pertinente, cercana al contexto y a las condiciones que rodean el acto del cuidado.

La determinación del tiempo requerido para cumplir las demás funciones establecidas para el equipo de enfermería es un proceso complejo que necesita, de forma objetiva, la sistematización en la cotidianidad donde se ejerce el cuidado.

El análisis periódico del tiempo requerido por el personal de enfermería en las instituciones de salud, las organizaciones sociales y en programas con los grupos debe estar sustentado en un sistema de información oportuno y válido que identifique las acciones y el tiempo que exigen las categorías reguladas en el país.

\section{Financiamiento}

Universidad de Antioquia, con el tiempo de docencia para la práctica académica.

\section{Conflictos de interẽs}

Los autores declaran no tener conflicto de intereses.

\section{Referencias}

1. Consejo Internacional de Enfermeras. Personal fiable vidas salvadas [internet]; 2006 [citado 2006 may 13]. Disponible en: http://www.icn. ch/indkit2006sp.pdf

2. Ley 266 de 1996/25 de enero, por la cual se reglamenta la profesión de enfermería en Colombia y se dictan otras disposiciones [internet]. 
Diario Oficial 42.710. Disponible en: http://www.mineducacion.gov. co/1759/articles-105002_archivo_pdf.pdf

3. Ley $911 / 2004$ de 5 de octubre, por la cual se dictan disposiciones en materia de responsabilidad deontológica para el ejercicio de la profesión de enfermería en Colombia; se establece el régimen disciplinario correspondiente y se dictan otras disposiciones. Diario Oficial 45.693 de 06-10-2004.

4. Aiken LH, Sermeus W, Van den Heede K, Sloane DM, Busse R, McKee $M$, et al. Patient safety, satisfaction, and quality of hospital care: cross-sectional surveys of nurses and patients in 12 countries in Europe and the United States. BMJ. 2012;344:e1717. doi: http://dx.doi. org/10.1136/bmj.e1717

5. Aiken LH, Clarke SP, Sloane DM, Sochalski J, Silber JH. Hospital nurse staffing and patient mortality, nurse burnout and job dissatisfaction. J Ame Nurs Asso. 2002;288(16):1987-93.

6. Aiken LH, Sloane DM, Bruyneel L, Van den Heede K, Sermeus W, and the RN4CAST Consortium. Nurses' reports of working conditions and hospital quality of care in 12 countries in Europe. Int J Nurs Stud. 2013;50:143-53.

7. Aiken LH, Sloane DM, Bruyneel L, Van den Heede K, Griffiths P, Busse $\mathrm{R}$, Sermeus W. Nurse staffing and education and hospital mortality in nine European countries: A retrospective observational study. The Lancet. 2014. doi:10.1016/S0140-6736(13)62631

8. Decreto $1011 / 2006$, de 3 de abril, por el cual se establece el Sistema Obligatorio de Garantía de Calidad de la Atención de Salud del Sistema General de Seguridad Social en Salud. Diario Oficial 46.230 de 03-04-2006.

9. Martín RA. Burnout en enfermería: prevalencia y factores relacionados en el medio hospitalario. Revista Científica de la Sociedad Española de Enfermería Neurológica. 2015;41(1):9-14.

10. Puerta Cataño AM, Giraldo Orozco A, Mosquera Soto HD, Preciado Arango L, Tabares Graciano D. Diagnósticos de enfermería más frecuentes en el servicio de hospitalización en la ESE Hospital Primer Nivel. Medellín: Facultad de Enfermería Universidad de Antioquia; 2010.

11. Puerta Cataño AM, Lopera CR, Macías PJ, Montenegro SL, Sánchez RD. Tiempo requerido para las intervenciones en el cuidado enfermero, desde los diagnósticos de enfermería de algunos pacientes internados en la ESE Hospital Primer Nivel. Medellin: Facultad de Enfermería Universidad de Antioquia; 2012.

12. Puerta Cataño AM, Pérez SS. Tiempo requerido por día para la gestión del cuidado y para las intervenciones del cuidado, desde el Proceso de Atención de Enfermería, de algunos pacientes internados en un Primer Nivel. Medellín: Facultad de Enfermería Universidad de Antioquia; 2012.

13. Puerta Cataño AM, Buitrago BL, Gallego RD. Tiempo requerido por categoría de personal de enfermería para realizar funciones de apoyo al cuidado y para el funcionamiento del servicio de hospitalización. ESE 
Hospital Primer Nivel. Medellin: Facultad de Enfermería Universidad de Antioquia; 2013.

14. Puerta Cataño AM, Montoya VJ, Valencia SY. Tiempo requerido de profesional de enfermería para realizar algunas funciones relacionadas con la gestión del servicio de hospitalización, investigación y educación. ESE Hospital Primer Nivel. Medellin: Facultad de Enfermería Universidad de Antioquia; 2013.

15. Puerta Cataño AM, Arroyave PA, Botero SM, Carvajal H, Cano MJ. Tiempo de personal de enfermería para realizar un cuidado con calidad y todas las funciones relacionadas con investigación, gestión del cuidado, educación y gestión del servicio de hospitalización. ESE Hospital Primer Nivel. Medellin: Facultad de Enfermería Universidad de Antioquia; 2013.

16. Puerta Cataño AM. Planes de dotación desde el cuidado, una utopía alcanzable. Documento procedente del XIV Congreso Colombiano de Enfermeria; Manizales, Colombia; julio de 1998.

17. Whetsell M. La investigación en enfermería. Documento procedente de la Celebración 60 años Facultad de Enfermería, Universidad de Antioquia, Medellín, Colombia; 2010.

18. Carrillo González GM, Rubiano Mesa YL. La investigación en validación de diagnósticos de enfermería. Rev Cubana Enfermer [internet]. 2007 Sep [citado 2016 mar 09];23(3). Disponible en: http://scielo.sld.cu/scielo.php?script $=$ sci_arttext\&pid=S0864-03192007000300009\&lng $=$ es

19. Miranda NR, Rijk A, Schaufeli W, Iapichino G. Nursing activities score. Crit Care Med. 2003; Crit Care Med. 2003 Feb;31(2):374-82.

20. Grillo Padilhaa K, Ferreira Queijoa A, Reis Miranda D. Nursing activities score in the intensive care unit: analysis of the related factors. Intensive Crit Care Nurs. 2008 Jun;24(3):197-204.

21. Conishi GR. Nursing Activities Score (NAS) como instrumento para medir carga de trabalho de enfermagem em UTI adulto. Rev Esc Enferm USP. 2007;41(3):346-54.

22. Benner P. From novice to expert: excellence and power in clinical nursing practice. Am J Nurs. 1982 Mar;82(3):402-7.

23. Leininger M. Transcultural nursing: Concepts, theories and practices. Columbus, OH: Greyden; 1994. 
